\title{
Factors affecting Embryo Quality and Pregnancy Outcome in Intra-Cytoplasmic Sperm Injection using Decapeptyl and Cetrorelix- A retrospective study
}

\author{
Laiba Masood', Faiza Alam², Nida Zahid³, Ahmed Ayaz', Syed Sajjad Hussain³, Rehana Rehman+4 \\ ${ }^{1}$ Medical College, Aga Khan University, Karachi. \\ 2PAPRSB Institute of Health Sciences, Universiti Brunei Darussalam, Bandar Seri Begawan, Brunei. \\ ${ }^{3}$ Australian Concept Infertility Medical Center, Rojhan Street, Clifton, Karachi, Pakistan. \\ ${ }^{4}$ Department of Biological and Biomedical Sciences, Aga Khan University, Stadium Road, Karachi, 74800, Pakistan.
}

\begin{abstract}
Objective: To compare embryo quality and pregnancy outcome of patients undergoing Intra-Cytoplasmic Sperm Injection (ICSI) via agonist and antagonist protocol at Australian Concept Infertility Medical Center (ACIMC), Karachi, Pakistan. Method: The ACIMC database extracted information of all adult infertile female patients who were recommended treatment of ICSI between $1^{\text {st }}$ June 2015 till $31^{\text {st }}$ August 2017.They were categorized on the basis of treatment by gonadotropin releasing hormone. One group was treated with the agonist (long term) and the other with the antagonist. The data was analyzed on STATA 12, independent T test/ Mann-Whitney U test, chi-square/ fisher exact test, and linear regression were performed where appropriate. Results: Of the 267, patient, 222 received $\mathrm{GnRH}$ agonists, while 45 were given GnRH antagonist. Univariable analysis showed an association of good quality embryos with the GnRH antagonist protocol, age, antral follicles, number of eggs fertilized and levels of; estradiol, follicle stimulating hormone (FSH) and prolactin $(p<0.25)$. On the multivariable analysis, prolactin levels, number of eggs fertilized and inseminated were associated with good quality embryos. There was a significant relationship between estradiol and no of follicles ( $p$ value $<0.05)$. Positive pregnancy outcome correlated with age, follicle count, FSH levels and eggs inseminated $(\mathrm{p}<0.25)$. Conclusion: Our results show that treatment type doesn't have an influence on the embryo and pregnancy outcome. However, estradiol levels, prolactin levels, number of follicles and particularly female age are predictors of embryo quality.
\end{abstract}

Keywords: intra-cytoplasmic sperm injection, treatment protocols, GnRH agonist, GnRH antagonist and pregnancy outcome

\section{Introduction}

The pulsatile secretion of Gonadotropin Releasing Hormone $(\mathrm{GnRH})$ in the natural cycle leads to a single follicle being developed and released. In Controlled Ovarian Stimulation (COS), multiple follicles are produced, which is an integral part of intra-cytoplasmic sperm injection (ICSI). ${ }^{1}$ In order to regulate ovulation in patients undergoing exogenous stimulation of the ovaries, it is important to keep the luteinizing hormone (LH) at an optimum level to prevent premature luteinization and ovulation. ${ }^{2}$ This is made possible by down-regulation of ovaries with exogenous administration of GnRH analogues. These analogues include both agonists and antagonist.

$\mathrm{GnRH}$ agonists suppress the release of the gonadotropins follicle stimulating hormone (FSH) and luteinizing hormone (LH) by desensitizing pituitary receptors, a phenomenon called "down-regulation". This treatment starts either on day 21 of the previous cycle (long protocol) or after the start of the period (short or flare-up protocol). However, both the long and short protocol have been shown to yield similar results. ${ }^{3} \mathrm{GnRH}$ agonist use has been associated with increased

Financial support: Department of Biological \& Biomedical Sciences.

Conflict of interest: Authors declare no conflict of interest.

Submitted: $14 / \mathrm{Sep} / 2020$

Accepted: 04/Mar./2021

Study was carried out at Australian Concept Infertility Medical Centre.

Copyright Masood et al. This is an Open Access article distributed under the terms of the Creative Commons Attribution License, which permits unrestricted use, distribution, and reproduction in any medium, provided the original work is properly cited. 
estradiol (E2) levels, improved oocyte yield, and higher fertilization and pregnancy rates. However, this method has the potential to cause ovarian over suppression, leading to decreased follicles and an increased need for gonadotropins. ${ }^{4,5}$

On the other hand, GnRH antagonists act by blocking the release of pituitary gonadotropins by competitive blockade of GnRH-receptors. ${ }^{6}$ The extent of receptor blockade depends on the dose of antagonist and is reversible on treatment cessation. Antagonists are administered in the mid-cycle and prevent a premature LH surge or ovarian over-suppression, thus maintaining normal follicular maturation. ${ }^{7}$ A shorter duration of stimulation, reduced gonadotropin consumption and prevention of ovarian hyper-stimulation are some advantages associated with antagonist treatment. ${ }^{2}$

Comparison of GnRH protocols shows that while agonists prevent premature follicle luteinization (PL), antagonist treatment may lead to reduced endometrial growth factors, and poorer endometrial receptivity. ${ }^{2}$ However, evidence regarding pregnancy outcomes in $\mathrm{GnRH}$ agonist versus antagonist protocols is inconclusive, with some studies reporting better outcome with agonist treatment and others with antagonist. ${ }^{7,8}$ Moreover, the impact of antagonist treatment on oocyte maturation and development is also unclear. ${ }^{9}$

Apart from treatment modalities, certain individual factors also influence the outcomes of ICSI. These include BMI, age, hormone levels, follicular count and semen characteristics. ${ }^{10-14}$ Age and BMI have shown to interfere with the regular menstrual cycle and hormone regulation in females. High FSH levels have shown an association with increased pregnancy rates. ${ }^{11}$ These characteristics are important to analyze as they predict positive outcomes early in the treatment cycle.

Therefore, further investigation on this topic is essential to determine the most appropriate treatment protocol for individuals who wish to undergo ICSI. It is also essential to understand factors influencing outcomes to refine treatment modalities. Helping patients achieve a successful pregnancy in the least number of stimulated cycles is a persistent challenge for practitioners. An estimated prediction of results can also avoid the financial and emotional loss of the patients and their families. Thus, the objective of our study was to compare differences in embryo quality and pregnancy outcome in females undergoing treatment with agonist and antagonist protocols.

\section{Materials and Methods}

A retrospective study was carried out from June 2015 till August 2017 in Australian Concept Infertility Medical Centre (ACIMC) after ethical approval (2018-0308-335).

'Patient and public involvement'; It was a retrospective study hence patients were not involved directly. The data of females who received agonist $(n=220)$ and $(n=45)$ antagonist protocol was collected on the basis of female age range between 20 and 40 years, who have been recommended treatment of ICSI due to infertility by: male factor (e.g. varicocele, prior surgeries and semen abnormalities), female factor (e.g. polycystic ovaries, uterine fibroids, endometriosis and tubal blockade etc.) or unexplained cause to achieve pregnancy. Females with thyroid dysfunction and abnormal prolactin levels were excluded. Couples with male factors causing infertility were also excluded. Moreover, females who developed Ovarian Hyperstimulation syndrome (OHSS) and those whose treatment was discontinued, were also not included in the study. South Asian criteria for BMI i.e. (BMI $<23 \mathrm{~kg} / \mathrm{m}^{2}$ - normal, $23-24.9 \mathrm{Kg} / \mathrm{m}^{2}$ - overweight and $>25 \mathrm{Kg} / \mathrm{m}^{2}$ - obese) was employed to categorize the females ${ }^{12}$

The treatment protocol is elaborated in Figure 1 and 2. In both protocols, there was routine monitoring of patients via trans-vaginal sonography (TVS) and hormonal profiling of FSH, LH, estrogen and progesterone levels of patients. ICSI was

Agonist Protocol

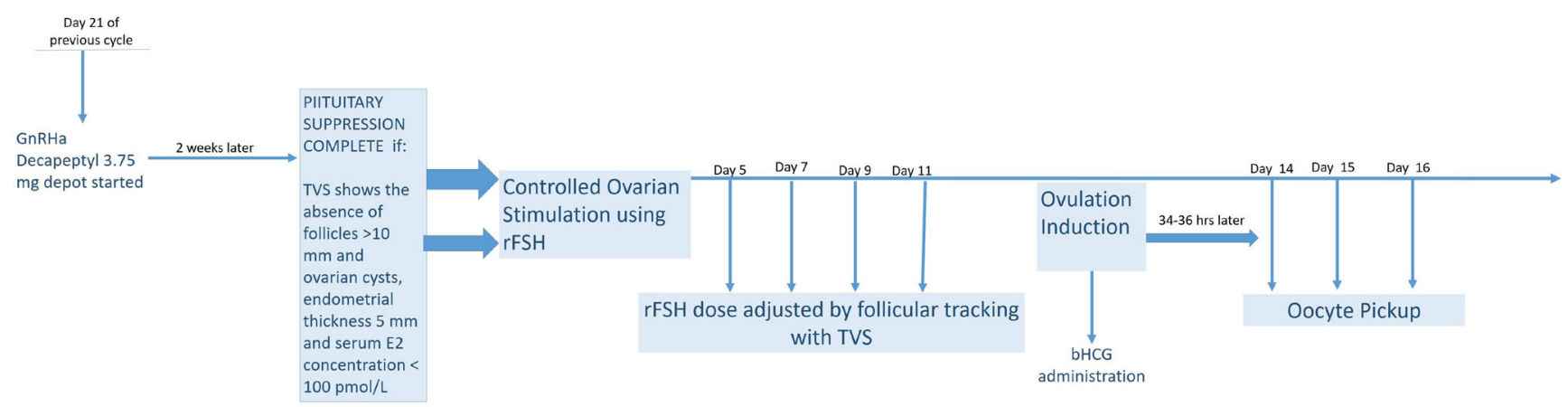

Figure 1: GnRH Agonist Protocol: GnRH agonist (Decapeptyl) $3.75 \mathrm{mg}$ started on the $21^{\text {st }}$ day of the luteal phase of the previous cycle and continued till 2 weeks until the pituitary suppression is confirmed as per the criteria by transvaginal ultrasound. Ovary Stimulation started using rFSH (dose....) with dose adjustment according to the follicular size. Ovulation is induced with beta HCG (dose) followed by oocyte retrieval on Day 14 after $34-36$ hours 


\section{Antagonist Protocol}

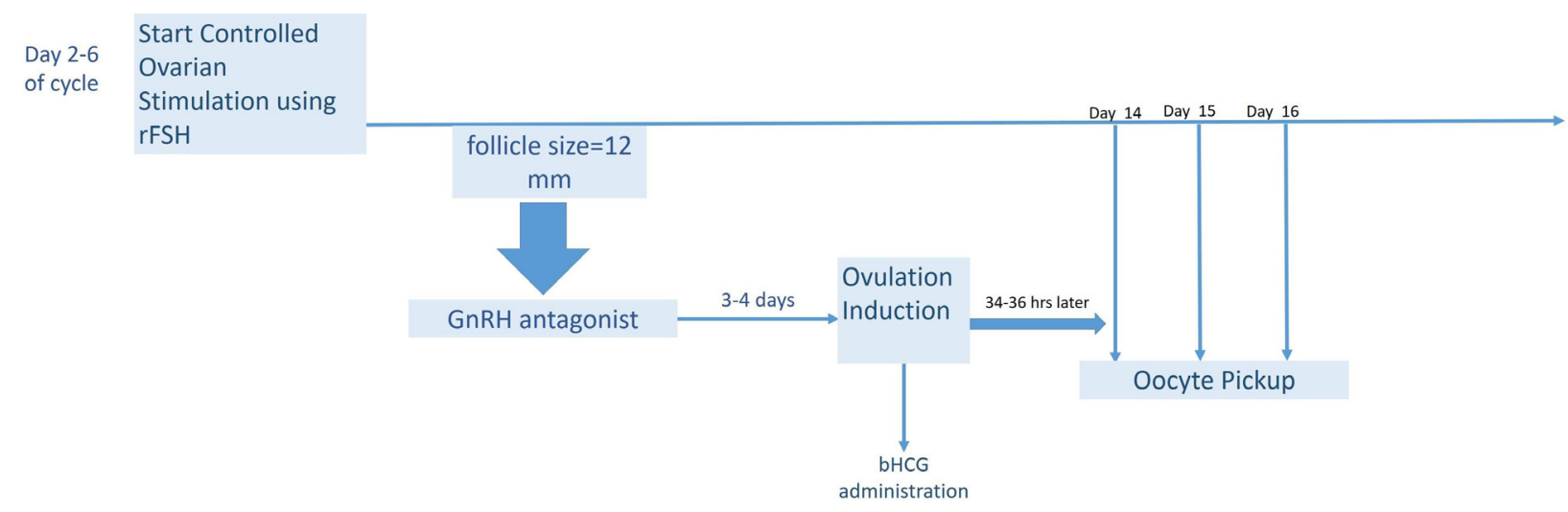

Figure 2: Treatment Protocol of Intracytoplasmic Sperm Injection with Antagonist

performed 3-6 h after oocyte recovery on all morphologically intact eggs in Metaphase II stage in labeled microinjection dish by procedure described by Rehman et al. ${ }^{13}$ Embryos were graded on the basis of cleavage rate and differentiation before embryo replacement and were transferred on the $2^{\text {nd }}$ or $3^{\text {rd }}$ or day after collection. ${ }^{14}$

Result were categorized on the basis of $\beta$-hCG concentrations performed 14 days after egg collection and ultrasound confirmation of an intrauterine gestational sac 14 days after results of beta hCG. Non-pregnant group had beta HCG $<25 \mathrm{mlU} / \mathrm{ml}$, and pregnant group had beta HCG > $25 \mathrm{mlU} / \mathrm{ml}$, an intrauterine gestational sac with cardiac activity confirmed by TVS.

\section{Statistical Analysis}

The data was analyzed on STATA 12. Descriptive for quantitative variables were reported as Mean \pm SD/ Median (IQR) and were assessed by independent $T$ test/ Mann-Whitney $U$ test where appropriate. The qualitative variables were reported as frequency and percentages and were assessed by chi-square/ fisher exact test. To assess relationship of retrieval of good quality embryos with type of treatment and other factors, linear regression was performed and unadjusted and adjusted beta coefficient with $95 \% \mathrm{Cl}$ was reported. To assess the relationship of pregnancy outcome with type of treatment and other factors, Cox regression analysis was performed and unadjusted and adjusted relative risk (RR) with 95\% Cl was reported. All plausible interactions and confounding were assessed. A p-value of $<0.05$ was considered significant.

\section{Results}

Table 1 indicates comparison of the demographics, according to the type of treatment received by females undergoing ICSI. 45 females received antagonist treatment while 222 received agonist. We observed that mean age and BMI of females was not significantly different in both the treatment groups.

Table 2 shows the hormonal levels, number of follicles, eggs and embryo according to the type of treatment received. The median estradiol level was significantly higher among females who were treated with agonist versus antagonist $(p<0.001)$. However, we did not find any significant difference in FSH, LH, Prolactin, TSH and AMH levels between the two treatment groups. The median number of follicles was significantly higher among females who received agonist treatment as compared to those who received antagonist treatment $(p=0.03)$. However, we did not find any significant difference in the median number of antral follicles, median no of eggs fertilized and inseminated between the two treatment groups. The median number of poor-quality embryos was significantly higher among females who received agonist treatment versus those who received antagonist treatment $(p=0.04)$. A higher proportion of females who were treated with antagonist conceived successfully as compared to those who received agonist, however this difference was not statistically significant $(p=0.31)$.

Table 3 depicts, univariate analysis to determine association of type of treatment and other factors with retrieval of good quality embryos among females undergoing ICSI. 
Table 1: Demographics of participants according to the type of treatment received

\begin{tabular}{|c|c|c|c|}
\hline \multirow{2}{*}{ Variables } & \multicolumn{2}{|c|}{ Type of treatment } & \multirow{2}{*}{ P value } \\
\hline & Antagonist $(n=45)$ Mean \pm SE $/ n(\%)$ & Agonist $(n=222)$ Mean $\pm S E /(n(\%)$ & \\
\hline \multicolumn{4}{|l|}{ Demographics } \\
\hline Age (in years) & $32.80 \pm 0.89$ & $32.77 \pm 0.353$ & 0.97 \\
\hline Height (in cms) & $154.47 \pm 0.872$ & $153.84 \pm 0.427$ & 0.54 \\
\hline Weight (Kgs) & $71.38 \pm 1.860$ & $67.76 \pm 0.971$ & 0.12 \\
\hline BMI (in kg/m²) & $30.11 \pm 0.837$ & $28.40 \pm 0.400$ & 0.08 \\
\hline \multicolumn{4}{|l|}{ BMI (in kg/m²) } \\
\hline$<18$ underweight & $0(0 \%)$ & $2(0.9 \%)$ & 0.92 \\
\hline 18-22.9 normal weight & $4(8.9 \%)$ & $24(11.1 \%)$ & \\
\hline 23-24.99 overweight & $5(11.1 \%)$ & $29(13.4 \%)$ & \\
\hline$\geq 25$ obese & $36(80 \%)$ & $161(74.5 \%)$ & \\
\hline
\end{tabular}

Table 2: Hormonal levels, no of follicles, eggs and embryo according to the type of treatment received by participants

\begin{tabular}{|c|c|c|c|}
\hline \multirow{2}{*}{ Variables } & \multicolumn{2}{|c|}{ Type of treatment } & \multirow[b]{2}{*}{ P value } \\
\hline & $\begin{array}{c}\text { Antagonist }(n=45) \text { Mean } \pm \text { SE /Median } \\
\text { (IQR) }\end{array}$ & $\begin{array}{l}\text { Agonist ( } n=222) \text { Mean } \pm \text { SE /Median } \\
(\text { IQR) }\end{array}$ & \\
\hline \multicolumn{4}{|l|}{ Hormones } \\
\hline Estradiol (mIU/ml) & $3600(2200-5500)$ & $5500(3200-8200)$ & $<0.001^{*}$ \\
\hline $\mathrm{FSH}(\mathrm{mlU} / \mathrm{ml})$ & $7.36 \pm 0.595$ & $7.39 \pm 0.326$ & 0.97 \\
\hline $\mathrm{LH}(\mathrm{mlU} / \mathrm{ml})$ & $5.00(3.00-8.00)$ & $5.21(3.85-8.00)$ & 0.15 \\
\hline Prolactin (mIU/L) & $19.00(12.00-25.00)$ & $15.00(10.00-22.00)$ & 0.09 \\
\hline \multicolumn{4}{|l|}{ Follicles and eggs } \\
\hline No of Follicles & $10.00(6.00-15.50)$ & $13.00(7.00-20.00)$ & $0.03 *$ \\
\hline No of Eggs Inseminated & $5.00(3.00-8.00)$ & $6.00(3.00-10.00)$ & 0.24 \\
\hline No of Eggs Fertilized & $5.00(2.00-7.00)$ & $5.00(2.00-9.00)$ & 0.5 \\
\hline \multicolumn{4}{|l|}{ Embryo quality } \\
\hline No of Poor quality & $1.00(0.00-3.00)$ & $2.00(1.00-4.00)$ & $0.04 *$ \\
\hline \multicolumn{4}{|l|}{ Pregnancy } \\
\hline Yes & $16(35.6 \%)$ & $62(27.9 \%)$ & \multirow{2}{*}{0.31} \\
\hline No & $29(64.4 \%)$ & $160(72.1 \%)$ & \\
\hline
\end{tabular}

*significant at $\mathrm{p}$ value $<0.05$ by using Mann-Whitney $U$ test

We observed a significant association quality of embryos with the type of infertility treatment at $p$ value $<0.25$. There was a higher expected retrieval of good quality embryos among females treated with agonist as compared to those who were treated with antagonist.

The retrieval of good quality embryos was expected to decrease by 0.14 units with every 1 -year increase in age. However, there was no significant association of retrieval of good quality embryos with BMI and semen parameters. We observed that the expected retrieval of good quality embryos was 0.21 units higher with every 1 unit increase in the number of follicles. Similarly, with every 1-unit increase in number of right and left antral follicles the expected retrieval 
Table 3: Univariate analysis to determine association of treatment with good quality of embryos among females undergoing ICSI

\section{Variables}

\section{Type of treatment}

Antagonist (ref)

Agonist

\section{Demographics}

Age years

No of eggs Inseminated

Hormones

Estradiol

$0.0004(0.00003)$

$\mathrm{FSH}$

$-0.17(0.03)$

LH

0.007 (0.004)

Prolactin
$95 \% \mathrm{Cl}$

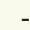

$-0.15,1.64^{\star}$

$-0.21,-0.08^{*}$

$0.19,0.23$ *

$0.20,0.33^{*}$

$0.20,0.34$ *

$0.45,0.52 *$

$0.40,0.46 *$

$0.0003,0.0005 *$

$-0.24,-0.10$ *

$-0.02,0.08$

$-0.001,0.01$ *

nalysis; Where FSH: follicle stimulating hormone, LH: Leutinizing hormone, TSH: thyroid *significant at $\mathrm{p}$ value $<0.25$ by univariate linear re
stimulating hormone, $\mathrm{AMH}$ : anti-mullerian hormone

of good quality embryos was increased by 0.26 and 0.27 units respectively. Moreover, with every 1 unit increase in the number of eggs fertilized and inseminated the retrieval of good quality embryo was expected to increase by 0.49 and 0.43 units respectively. The hormones (estradiol, FSH and prolactin) also had a significant association with retrieval of good quality embryos. With every 1 unit increase in estradiol and prolactin the expected retrieval of good quality embryos was increased by 0.0004 and 0.007 respectively, however, it was decreased by $1 \mathrm{mIU} / \mathrm{ml}$ increase in FSH. There was no significant association of LH, TSH and AMH with embryo quality.

Table 4 indicates multivariable analysis to determine association of type of treatment with retrieval of good quality embryos among females undergoing ICSI.

With every 1-unit increase in \ prolactin levels, the expected good quality embryo retrieval was increased by 0.006 units. Similarly, the retrieval of good quality embryos was expected to increase by 0.25 and 0.13 units with 1 -unit increase in no of eggs fertilized and inseminated respectively. There was significant interaction between estradiol and no of follicles.

Table 5 shows univariable analysis to determine association of type of treatment and other factors with pregnancy outcome among females undergoing ICSI. We observed that there was no significant association of type of treatment and pregnancy outcome. However, with every 1-unit increase in age the chance of pregnancy was decreased significantly by $6.6 \%$. Moreover, the chance of pregnancy was increased by; 1.035 with every 1-unit increase in the follicle count, by 1.061 times with increase in number of follicles and by 1.055 times with increase in number of eggs inseminated. The likelihood of pregnancy was decreased by 6.85 with every $1 \mathrm{mIU} / \mathrm{ml}$ increase in FSH levels. However, the chance of pregnancy was significantly increased by almost 12\% with increase in the yield of good quality embryos.

No significant association was evident of semen parameters, BMI, hormones such as AMH, LH, TSH and prolactin with pregnancy outcome.

Table 6 indicates multivariable analysis to determine association of type of treatment and other factors with pregnancy outcome among females undergoing ICSI.

After adjusting for all the covariates, we observed that there was no significant association of type of treatment i.e. antagonist versus agonist with pregnancy outcome. However, the chance of pregnancy was significantly decreased by $5.1 \%$ with every 1 -year increase in age. The likelihood of pregnancy was increased by 1.048 times (95\% Cl 1.010-1.088) with every one-unit increase in the number of eggs fertilized. 
Table 4: Multivariate analysis to determine association of type of treatment and other factors with retrieval of good quality embryos among females undergoing ICSI

\section{Variables}

\section{Type of Treatment}

Antagonist (ref)

Agonist

Estradiol

Prolactin

No of Follicles

No of eggs Fertilized

No of eggs Inseminated

Estradiol * no of follicles
Beta Coefficient

$95 \% \mathrm{Cl}$

1

$0.16(0.22)$

$-0.000002(0.00004)$

0.006(0.001)

0.005(0.021)

$0.25(0.05)$

0.13(0.05)

$0.000004(0.000002)$
$-0.27,0.59$

$-0.0001,0.0001$

$0.003,0.01^{*}$

$-0.04,0.05$

$0.17,0.35 *$

$0.03,0.23 *$

$0.0000004-0.000007^{* \star}$

*significant at $p$ value $<0.05$ by multivariable linear regression analysis; **significant interaction at $p$ value $<0.05$

Table 5: Univariate analysis to determine association of type of treatment and other factors with pregnancy outcome among females undergoing ICSI

Variables

Type of treatment

Antagonist (ref)

Agonist

\section{Demographics}

Age years

1.035

1.053

1.068

1.061

1.055

1.000

0.932

1.119

1.075

1.043

No of Poor quality Median (IQR)
$95 \% \mathrm{Cl}$

$0.453-1.361$

$0.897-0.973 *$

1.018-1.053*

1.012-1.095*

1.028-1.109*

1.025-1.099*

$1.024-1.088^{*}$

$0.000001-0.0001$ *

0.861-1.009*

1.053-1.188*

1.018-1.137*

1.004-1.084*

*significant at $p$ value $<0.25$ by univariate cox regression analysis; Where FSH: follicle stimulating hormone, LH: luteinizing hormone, TSH: thyroid stimulating hormone, AMH: anti-mullerian hormone

\section{Discussion}

The comparative effectiveness of GnRH agonist and antagonist treatment protocols for assisted reproductive technology (ART) is still under debate. ${ }^{5}$ We conducted this study in order to assess the differences in outcomes of the two protocols, and the factors affecting pregnancy rates and embryo quality. We found that $\mathrm{GnRH}$ agonist treatment was associated with 
Table 6: Multivariable analysis to determine association of type of treatment and other factors with pregnancy outcome among females undergoing ICSI

\section{Variables}

$$
\text { Relative risk (RR) }
$$

p-value

\section{Type of treatment}

\begin{abstract}
Antagonist (ref)
\end{abstract}
Agonist

0.751

0.949

Age

1.048
$0.432-1.308$

0.909-0.990

1.010-1.088
0.312

$0.016^{*}$

0.014 *

Log likelihood: -421.102 ; *significant at $p$ value $<0.05$ by multivariable cox regression analysis

a significantly higher number of poor-quality embryos. However, we found no other statistically significant differences in either of the two protocols. Embryo outcomes and pregnancy outcomes were affected by estradiol levels, prolactin levels and age.

Our study shows the retrieval of good quality embryos to be greater in the antagonist group, compared to the agonist group. This was seen on univariable analysis, but not on multivariable analysis. Moreover, GnRH agonist treatment was found to be associated with a significantly higher number of poor-quality embryos. Varying results are seen in international literature. More mature oocytes of better quality were observed in the $\mathrm{GnRH}$ antagonist treatment groups in several studies. ${ }^{15}$ Conversely, in a prospective randomized controlled trial Olivennes et al. reported oocyte quantity and and embryo quality to decrease with the use of GnRH antagonist. ${ }^{16}$ Difference could be accounted by the use of different regimens of drugs to carry out the treatment. In our study, the GnRH agonist, Decapeptyl was used and administered before rFSH. For GnRH antagonist protocol, Cetrorelix was used after exogenous rFSH administration. De Placido et al. and Olivennes et al. used the same regimen as ours, however, Malmusi et al., used Ganirelix as the antagonist, but the rest of the procedure was the same. ${ }^{5,15,16}$ Schmidt et al., reported Leuprolide use as an agonist compared with Ganirelix as the antagonist, and found no statistical difference in the embryo outcome. ${ }^{17}$ Since GnRH antagonists have a direct effect on the steroid production in the granulosa cells of the follicles, a better quality and more mature embryo could be attributed to this treatment protocol. ${ }^{18}$

There was no association between treatment type and pregnancy outcomes. Studies in other populations have shown a higher pregnancy rate in patients undergoing the antagonist treatment Even in the poor responder's subgroup of patients, the pregnancy rate is higher in the antagonist group. ${ }^{19}$ In fact, Craft et al., reported higher pregnancy rates in patients undergoing antagonist treatment, who previously underwent the agonist treatment and did not respond. ${ }^{20}$ However, this study also reported greater cancellation rates in the antagonist protocol. According to Kolibianakis et al., however, live birth rate outcomes were not different in the two protocol. ${ }^{21}$

Basal estradiol and prolactin levels were significantly associated with retrieval of good quality embryos. Studies have shown similar results, where higher prolactin levels have led to better quality embryos being retrieved, along with higher pregnancy rates..$^{22}$ However, some studies have also shown no correlation between prolactin and the success of the treatments ${ }^{23}$

Prolactin receptors and its mRNA are present in mature oocytes, and have shown to play a role in oocyte maturation. ${ }^{23}$ Also, prolactin receptors have been found on the endometrium. They increase the endometrium's receptivity and enhances blastocyst implantation during artificial implantation. ${ }^{22}$

Furthermore, estradiol levels have been shown to have a positive impact on embryo and pregnancy outcomes in previous studies ${ }^{24}$ Estradiol has proved to slow the pace of follicular development and hence improve follicular synchronization. ${ }^{24,25}$ This could explain why embryo quality is improved by higher levels of estradiol. In fact, Estradiol levels are used to monitor follicles (along with trans vaginal ultrasound), and help in deciding when to give the hCG trigger for ovulation induction. ${ }^{26}$

Our results show no association of BMI with either of the treatment protocols, nor with the quality of embryos retrieved or with pregnancy rate. This is very different from most previous literature. Moragianni et al., suggested that patients with BMI higher than $30 \mathrm{~kg} / \mathrm{m}^{2}$ had a $68 \%$ lower chance of conceiving ${ }^{10}$ Studies have even suggested a disruption of the menstrual cycle and therefore negative ICSI outcomes in patients with a higher BMI. ${ }^{26} \mathrm{~A}$ recent systematic review from the Middlesex University, London shows a decreasing trend of pregnancy and live birth rates in patients with high BMI. ${ }^{26}$

However, a study by Nicholas et al., revealed that the number of oocytes and quality of embryo was not affected by BMI. Only clinical pregnancy outcomes were lower in patients with a higher BMI ${ }^{27}$ 
The retrieval of good quality embryos and the chance of pregnancy were expected to decrease with increasing age. This is supported by most studies, where maternal age was the determining factor of embryo quality, count and pregnancy outcomes. ${ }^{25}$ This could be attributed to uterine problems owing to advanced age, a disturbed hormonal environment leading to problems in ovulation, failure of embryo implanting, reduced ovarian reserve and poor oocyte quality. ${ }^{28}$

To the best of our knowledge, previous studies have focused primarily on the differences between the two GnRH treatment modalities, however, limited studies have explored the association of individual factors with positive pregnancy and embryo outcomes in patients undergoing treatment with GnRH protocols. Our study not only compares the patient characteristics and outcomes in the two treatment protocols, it also comments on the factors associated with positive outcomes.

Given the retrospective nature of this study, some limitations include the lack of randomization to GnRH treatment protocol. However, the patients in the agonist and antagonist treatment groups had similar baseline characteristics.

\section{Conclusion}

Our study has shown an association between GnRH antagonist treatment and good quality embryos, but without any significant difference in pregnancy outcomes among the two treatment protocols. We recommend that further research be done to investigate the effects of the antagonist protocol on conception. Since, estradiol and prolactin levels have shown to improve embryo quality, further investigation on their affect at the molecular level should be conducted.

\section{Limitations \& Strengths}

- Limitations include the lack of randomization to GnRH treatment protocol.

- Only the long protocol treatment group is included

- However, the patients in the agonist and antagonist treatment groups had similar baseline characteristics.

\section{References}

1. Kyrou D, Al-Azemi M, Papanikolaou EG, Donoso P, Tziomalos K, Devroey P, et al. The relationship of premature progesterone rise with serum estradiol levels and number of follicles in GnRH antagonist/recombinant FSH-stimulated cycles. Eur J Obstet Gynecol Reprod Biol. 2012;162(2):165-8. http://dx.doi.org/10.1016/j.ejogrb.2012.02.025. PMid:22425288.

2. Gordts S, Van Turnhout C, Campo R, Puttemans P, Valkenburg M, Gordts S. A prospective randomised study comparing a GnRHantagonist versus a $\mathrm{GnRH}$-agonist short protocol for ovarian stimulation in patients referred for IVF. Facts Views Vis ObGyn. 2012;4(2):82-7. PMid:24753894.

3. Ho C-H, Chen S-U, Peng F-S, Chang C-Y, Lien Y-R, Yang Y-S. Prospective comparison of short and long GnRH agonist protocols using recombinant gonadotrophins for IVF/ICSI treatments. Reprod Biomed Online. 2008;16(5):632-9. http://dx.doi.org/10.1016/ S1472-6483(10)60476-2. PMid:18492366.

4. Berin I, Stein DE, Keltz MD. A comparison of gonadotropin-releasing hormone (GnRH) antagonist and GnRH agonist flare protocols for poor responders undergoing in vitro fertilization. Fertil Steril. 2010;93(2):360-3. http://dx.doi.org/10.1016/j.fertnstert.2008.11.007. PMid:19131055.

5. Malmusi S, La Marca A, Giulini S, Xella S, Tagliasacchi D, Marsella T, et al. Comparison of a gonadotropin-releasing hormone (GnRH) antagonist and GnRH agonist flare-up regimen in poor responders undergoing ovarian stimulation. Fertil Steril. 2005;84(2):402-6. http://dx.doi.org/10.1016/j.fertnstert.2005.01.139. PMid:16084881.

6. Griesinger G, Felberbaum R, Diedrich K. GnRH-antagonists in reproductive medicine. Arch Gynecol Obstet. 2005;273(2):71-8. http://dx.doi.org/10.1007/s00404-005-0021-2. PMid:15991015.

7. Al-Inany HG, Youssef MA, Ayeleke RO, Brown J, Lam WS, Broekmans FJ. Gonadotrophin-releasing hormone antagonists for assisted reproductive technology. Cochrane Database of Syst Rev. 2016;11(5):CD001750.

8. Bodri D, Sunkara SK, Coomarasamy A. Gonadotropin-releasing hormone agonists versus antagonists for controlled ovarian hyperstimulation in oocyte donors: a systematic review and meta-analysis. Fertil Steril. 2011;95(1):164-9. http://dx.doi.org/10.1016/j. fertnstert.2010.06.068. PMid:20684954.

9. Takahashi K, Mukaida T, Tomiyama T, Goto T, Oka C. GnRH antagonist improved blastocyst quality and pregnancy outcome after multiple failures of IVF/ICSI-ET with a GnRH agonist protocol. J Assist Reprod Genet. 2004;21(9):317-22. http://dx.doi. org/10.1023/B:JARG.0000045470.68525.a4. PMid:15587144.

10. Moragianni VA, Jones SM, Ryley DA. The effect of body mass index on the outcomes of first assisted reproductive technology cycles. Fertil Steril. 2012;98(1):102-8. http://dx.doi.org/10.1016/j.fertnstert.2012.04.004. PMid:22584023.

11. Thum MY, Kalu E, Abdalla H. Elevated basal FSH and embryo quality: Lessons from extended culture embryos: raised FSH and blastocyst quality. J Assist Reprod Genet. 2009;26(6):313-8. http://dx.doi.org/10.1007/s10815-009-9313-y. PMid:19513823. 
12. Alam F, Memon AS, Fatima SS. Increased body mass index may lead to hyperferritinemia irrespective of body iron stores. Pak J Med Sci. 2015;31(6):1521-6. PMid:26870128.

13. Rehman R, Hussain Z, Zahir H, Khan R. Impact of peak/mid luteal estradiol on pregnancy outcome after intracytoplasmic sperm injection. J Pak Med Assoc. 2014;64(7):780-4. PMid:25255586.

14. Rehman R, Irfan T, Jawed S, Hussain M, Ali R. Embryo quality in intracytoplasmic sperm injection: A quasi experimental design in Pakistan. J Pak Med Assoc. 2018;68(10):1451-5. PMid:30317340.

15. De Placido G, Mollo A, Clarizia R, Strina I, Conforti S, Alviggi CJF. Gonadotropin-releasing hormone (GnRH) antagonist plus recombinant luteinizing hormone vs. a standard GnRH agonist short protocol in patients at risk for poor ovarian response. Fertil Steril. 2006;85(1):247-50. http://dx.doi.org/10.1016/j.fertnstert.2005.07.1280. PMid:16412769.

16. Olivennes F, Belaisch-Allart J, Emperaire JC, Dechaud H, Alvarez S, Moreau L, et al. Prospective, randomized, controlled study of in vitro fertilization-embryo transfer with a single dose of a luteinizing hormone-releasing hormone (LH-RH) antagonist (cetrorelix) or a depot formula of an LH-RH agonist (triptorelin). Fertil Steril. 2000;73(2):314-20. http://dx.doi.org/10.1016/ S0015-0282(99)00524-5. PMid:10685535.

17. Schmidt DW, Bremner T, Orris JJ, Maier DB, Benadiva CA, Nulsen JC. A randomized prospective study of microdose leuprolide versus ganirelix in in vitro fertilization cycles for poor responders. Fertil Steril. 2005;83(5):1568-71. http://dx.doi.org/10.1016/j. fertnstert.2004.10.053. PMid:15866609.

18. Mitwally MF, Casper RF. Effect of in vivo GnRH agonist and GnRH antagonist on hCG and insulin-stimulated progesterone production by human granulosa-lutein cells in vitro. J Assist Reprod Genet. 2002;19(8):384-9. http://dx.doi.org/10.1023/A:1016398405248 PMid:12182445.

19. Lainas TG, Sfontouris IA, Papanikolaou EG, Zorzovilis JZ, Petsas GK, Lainas GT, et al. Flexible GnRH antagonist versus flare-up GnRH agonist protocol in poor responders treated by IVF: a randomized controlled trial. Hum Reprod. 2008;23(6):1355-8. http:// dx.doi.org/10.1093/humrep/den107. PMid:18403419.

20. Craft I, Gorgy A, Hill J, Menon D, Podsiadly BJHR. Will GnRH antagonists provide new hope for patients considereddifficult responders' to GnRH agonist protocols? Hum Reprod. 1999;14(12):2959-62. http://dx.doi.org/10.1093/humrep/14.12.2959. PMid:10601078.

21. Kolibianakis E, Collins J, Tarlatzis B, Devroey P, Diedrich K, Griesinger G. Griesinger GJHru. Among patients treated for IVF with gonadotrophins and GnRH analogues, is the probability of live birth dependent on the type of analogue used? A systematic review and meta-analysis. Hum Reprod Update. 2006;12(6):651-71. http://dx.doi.org/10.1093/humupd/dml038. PMid:16920869.

22. Kamel A, Halim AA, Shehata M, AlFarra S, El-Faissal Y, Ramadan W, et al. Changes in serum prolactin level during intracytoplasmic sperm injection, and effect on clinical pregnancy rate: a prospective observational study. BMC Pregnancy Childbirth. 2018;18(1):141. http://dx.doi.org/10.1186/s12884-018-1783-4. PMid:29739353.

23. Lebedeva IY, Singina GN, Lopukhov AV, Shedova EN, Zinovieva NA. Prolactin and growth hormone affect metaphase-II chromosomes in aging oocytes via cumulus cells using similar signaling pathways. Front Genet. 2015;6:274. http://dx.doi.org/10.3389/ fgene.2015.00274. PMid:26379702.

24. Joo BS, Park SH, An BM, Kim KS, Moon SE, Moon HS. Serum estradiol levels during controlled ovarian hyperstimulation influence the pregnancy outcome of in vitro fertilization in a concentration-dependent manner. Fertil Steril. 2010;93(2):442-6. http://dx.doi. org/10.1016/j.fertnstert.2009.02.066. PMid:19394001.

25. Zhang W, Tian Y, Xie D, Miao Y, Liu J, Wang X. The impact of peak estradiol during controlled ovarian stimulation on the cumulative live birth rate of IVF/ICSI in non-PCOS patients. J Assist Reprod Genet. 2019;36(11):2333-44. http://dx.doi.org/10.1007/s10815019-01568-w. PMid:31485870.

26. Rottiers A, Dalewyn L, Somers S, Alper M, Sakkas D, Gerris J. Correlation between sonographic follow-up of follicular growth, serum and salivary estradiol in women undergoing controlled ovarian stimulation (IVF/ICSI). Facts Views Vis ObGyn. 2018;10(4):173-9. PMid:31367289.

27. Nichols JE Jr, Crane MM, Higdon HL, Miller PB, Boone WR. Extremes of body mass index reduce in vitro fertilization pregnancy rates. Fertil Steril. 2003;79(3):645-7. http://dx.doi.org/10.1016/S0015-0282(02)04807-0. PMid:12620460.

28. Ahmed M, Shareef O, Adam I, Rayis D. Maternal age and intracytoplasmic sperm injection outcome in infertile couples at Khartoum, Sudan. F1000 Res. 2015;4:4. http://dx.doi.org/10.12688/f1000research.7386.1. PMid:27347370.

\author{
*Correspondence: \\ Rehana Rehman \\ Department of Biological and Biomedical Sciences, Aga Khan University \\ National Stadium Rd, Aga Khan University Hospital \\ Karachi, Karachi City, Sindh, Zip Code 74800, Pakistan \\ Phone Number+92-030722227775 \\ drrehana7@gmail.com
}




\section{Author information}

LM: Year 4 Medical Student, Medical College, Aga Khan University, Karachi

FA: PhD scholar, Department of Physiology, University of Karachi, Main University Road, Karachi, Sindh 75270, Pakistan

NZ: Data Analyst, Australian Concept Infertility Medical Center, Rojhan Street, Clifton, Karachi, Pakistan.

AA: Year 5 Medical Student, Medical College, Aga Khan University, Karachi

SSH: Chief Executive officer, Australian Concept Infertility Medical Center, Rojhan Street, Clifton, Karachi, Pakistan.

RR: Associate Professor, Department of Biological and Biomedical Sciences, Aga Khan University, Stadium Road, Karachi, 74800, Pakistan.

\section{Author contributions}

LM, FA, NZ, AA, SSH, RR: All authors took part in submission of manuscript. 